\title{
Erratum: Eigenstate clustering around exceptional points [Phys. Rev. A 102, 032203 (2020)]
}

\author{
C. Yuce (1)
}

(Received 5 August 2021; published 7 September 2021)

DOI: 10.1103/PhysRevA.104.039901

In our recent paper, we made a numerical mistake when plotting Fig. 1(a). The correct figure is shown below. The system still shows the non-Hermitian skin effect and, hence, the conclusions from this figure remain the same as mentioned in the published paper. In addition, we correct two statements in our paper. The first one is in the Introduction section. The sentence "In Hermitian systems, all eigenstates are linearly dependent from each other and hence no eigenstate clustering occurs" should be changed. We, therefore, state that orthogonal eigenstates in Hermitian systems are linearly independent from each other, and, hence, no eigenstate clustering occurs. The second one is in Sec. II. We change the sentence "In Hermitian systems, fidelities between any two distinct eigenstates are always zero." The new sentence is as follows: Fidelities between two eigenstates of a Hermitian Hamiltonian corresponding to different eigenvalues are always zero. Finally, the following sentence in Sec. II "As opposed to the orthogonal eigenstates, nonorthogonal eigenstates are not linearly dependent" must be omitted. The main conclusions of our paper are not changed. We note that our machine learning algorithm works best for highly nonreciprocal tight-binding non-Hermitian Hamiltonians.

We would like to thank F. M. Fernandez from INIFTA for identifying them [1].

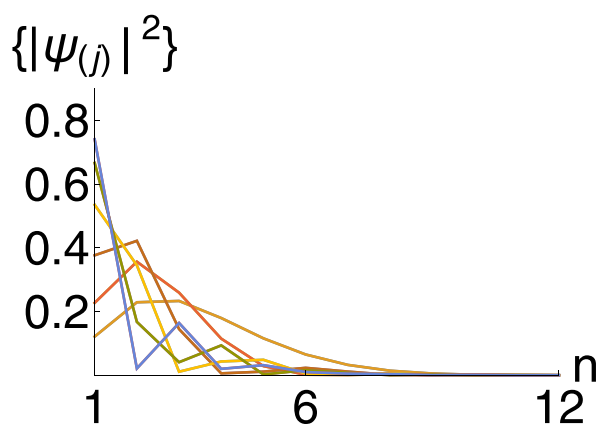

FIG. 1. Corrected version of the Fig. 1(a) in the published paper.

[1] F. M. Fernández, arXiv:2107.10674. 